\title{
Le cyberproduit
}

\author{
William Menvielle, Denis Pettigrew et Jocelyn D. Perreault ${ }^{1}$ \\ Université du Québec à Trois-Rivières
}

Dans son article Marketing on the Internet, Pallab mettait en avant le fait que le marketing se devait de s'adapter à ce médium révolutionnaire qu'est l'Internet. Bien que le marketing traditionnel ait encore de beaux jours devant lui, certains partisans du cybermarketing, cette intégration du marketing et de l'Internet, clament haut et fort «Le marketing classique est mort. Vive le marketing! ». Nous croyons que le marketing est pérenne, mais le but de notre interrogation est de voir comment il s'articule avec l'Internet.

Pour cela, nous définirons le cybermarketing, terme relativement récent apparu au milieu des années 1990, et nous nous intéresserons plus particulièrement au cybermarketing-mix, c'est-à-dire à l'agencement des célèbres «4 $\mathrm{P}$ » du marketing et des nouvelles technologies d'information et de communication (NTIC).

À cet égard, nous considérerons Internet sous trois aspects: comme un moyen de communication - c'est sans doute l'aspect le plus souvent évoqué -, comme un moyen de distribution pour télécharger des logiciels ou de la musique, et également comme un moyen de paiement, notamment lors de l'achat en ligne.

Cet article est la première réflexion d'une série portant sur chacune des variables du marketing-mix que sont le produit, le prix, la distribution et la communication. Dans ce premier article, nous nous intéresserons précisément au produit pour saisir comment il s'intègre à l'Internet. Nous considérerons de façon sous-jacente ce que les puristes nomment le cinquième $P$ du marketing-mix, à savoir la personnalisation du service ${ }^{3}$, qui consiste à garder un oil ouvert sur le client.

Notons finalement que notre approche se concentre uniquement dans une perspective de relation entreprise à consommateur (B2C), et que même si les relations entre entreprises présentent des volumes de transactions plus importants, les premières nécessitent plus d'efforts de la part des entreprises, soucieuses de trouver, par ce médium, de nouveaux débouchés.

Nous n'avons pas la prétention de proposer de nouveaux modèles de gestion ni un nouveau paradigme du marketing, mais simplement, à travers une revue de littérature et par une série d'exemples, nous mettrons en lumière les enjeux de cette nouvelle tendance, après avoir donné une définition du cybermarketing et du cybermix.

\section{Le cybermarketing}

Cronin ${ }^{4}$ est sans doute l'une des premières à s'être intéressée au concept de marketing lié à l'Internet. Dès 1995, l'auteure propose le terme intermarketing, néologisme résultant de la contraction des mots «Internet marketing » et «interactive marketing», et le définit comme étant l'utilisation du marketing sur Internet. La littérature recense également l'existence du terme e-marketing, provenant de « electronic marketing», par analogie aux différents mots composés selon ce modèle tels $e$-mail, $e$-cash, e-commerce.

Avant de donner une définition du «cybermarketing», terme relativement nouveau apparu dans la littérature de gestion il y a peu, il est intéressant de se pencher sur le préfixe «cyber» et de définir la cybernétique. Selon le dictionnaire Le Petit Robert, la cybernétique est la «science constituée par l'ensemble des théories relatives aux communications et à la régulation dans l'être vivant et la machine». Settles ${ }^{5}$ précise à cet égard que ce terme a une connotation virtuelle et qu'il s'est développé de concert avec la culture d'Internet au cours de ces der- 
nières années. Ainsi, un vocabulaire nouveau est apparu, intégrant le préfixe « cyber », comme dans cyberespace, cyberconsommateur, cybermonnaie et cybermarketing, qui désigne l'intégration de ces termes dans le monde virtuel. À titre d'exemple, un cyberconsommateur est un consommateur magasinant par Internet.

Keeler ${ }^{6}$ reprend donc le préfixe «cyber » pour former l'expression cybermarketing, qu'il définit de la façon suivante: «Cybermarketing means: using the power of online networks, computer communications, and digital interactive media to reach your marketing objectives. » Pour l'auteur, le cybermarketing est donc le marketing du cyberespace, ce monde virtuel né de la fusion de l'informatique et des télécommunications.

Le cybermarketing n'est toutefois pas étranger au marketing, dont il reprend les fondements, en demeurant centré sur le client et sa satisfaction. Pour Settles ${ }^{7}$, le cybermarketing comprend quatre dimensions, représentant les quatre enjeux majeurs de cette notion et permettant aux entreprises de :

- proposer une mise en marché des produits en respectant les souhaits des consommateurs;

- prospecter plus efficacement les clients ;

- proposer un service et un support plus efficaces et moins onéreux ;

- compléter le marketing traditionnel.

À ces quatre enjeux, l'auteur fait correspondre sept éléments pour rencontrer ces objectifs, qu'il énumère comme suit: 1) construire la reconnaissance et la loyauté d'une marque de commerce ; 2) élaborer des promotions en temps réel ; 3) dispenser de l'éducation sur le marché ; 4) élaborer un service de distribution et des démonstrations de produits; 5) établir des relations avec le public et la presse; 6) faire de la recherche; 7) faire du développement de produit, de service et de support.

Dans sa description des objectifs du cybermarketing, l'auteur reste centré sur la notion de client comme la cible du marketing. Les nouvelles technologies comme l'Internet ${ }^{8}$ ne sont pour lui qu'un moyen de communication nouveau comme la radio ou la télévision l'ont été bien avant, qui sert cependant également d'outil pour la distribution de certains produits pouvant être livrés par voie électronique (logiciels, musique, vidéos, documents écrits) et de support pour le paiement électronique.

\section{Le lien entre stratégie marketing et Internet est proposé par Dufour, qui donne sa définition du cybermarketing : «la branche du marketing qui analyse et met en œuvre une stratégie marketing grâce au réseau et aux outils Internet».}

Le lien entre stratégie marketing et Internet est proposé par Dufour 9 , qui donne sa définition du cybermarketing: «la branche du marketing qui analyse et met en œuvre une stratégie marketing grâce au réseau et aux outils Internet». Toutefois, l'auteur tient à souligner que le cybermarketing n'est «pas limité au réseau Internet, mais concerne également les futures autoroutes de l'information ou la télévision interactive». Pour notre part, nous soutenons également cette thèse et tenons à préciser que cette notion s'applique à de nombreux modèles d'affaires électroniques ${ }^{10}$ parmi lesquels on recense entre autres le commerce électronique et l'entreprise électronique.

Le cybermarketing s'appuie toutefois sur un élément essentiel du marketing : la notion d' «interactivité », bien représentée dans la technologie Internet et popularisée par Peppers et Rogers ${ }^{11}$ dans leur notion de «marketing one-to-one».

En se basant sur la définition de Kotler et autres ${ }^{12}$ du marketing-mix, reprenant les célèbres « $4 \mathrm{P} »$ de Mac Carthy (promotion, place, prix, produit), il est possible de composer le terme cybermarketing-mix ou cybermix, comme le louange Dufour ${ }^{13}$, pour désigner l'intégration d'Internet dans le marketing-mix d'une entreprise. Un cadre imagé est d'ailleurs présenté à la figure 1 suivante, qui prend en considération les éléments nouveaux auxquels le cybermarketing-mix doit s'intéresser pour intégrer les dimensions des NTIC.

Nous proposons donc une incursion dans ce cybermonde et invitons le lecteur à passer en revue les quatre composantes de ce nouveau mix. D'autres réflexions seront nécessaires ayant trait aux autres $« \mathrm{P} »$ afin de comprendre que certaines variables connaissent peu de modifications alors que d'autres subissent une profonde mutation. 


\section{Figure 1. La stratégie de cybermarketing de l'entreprise}

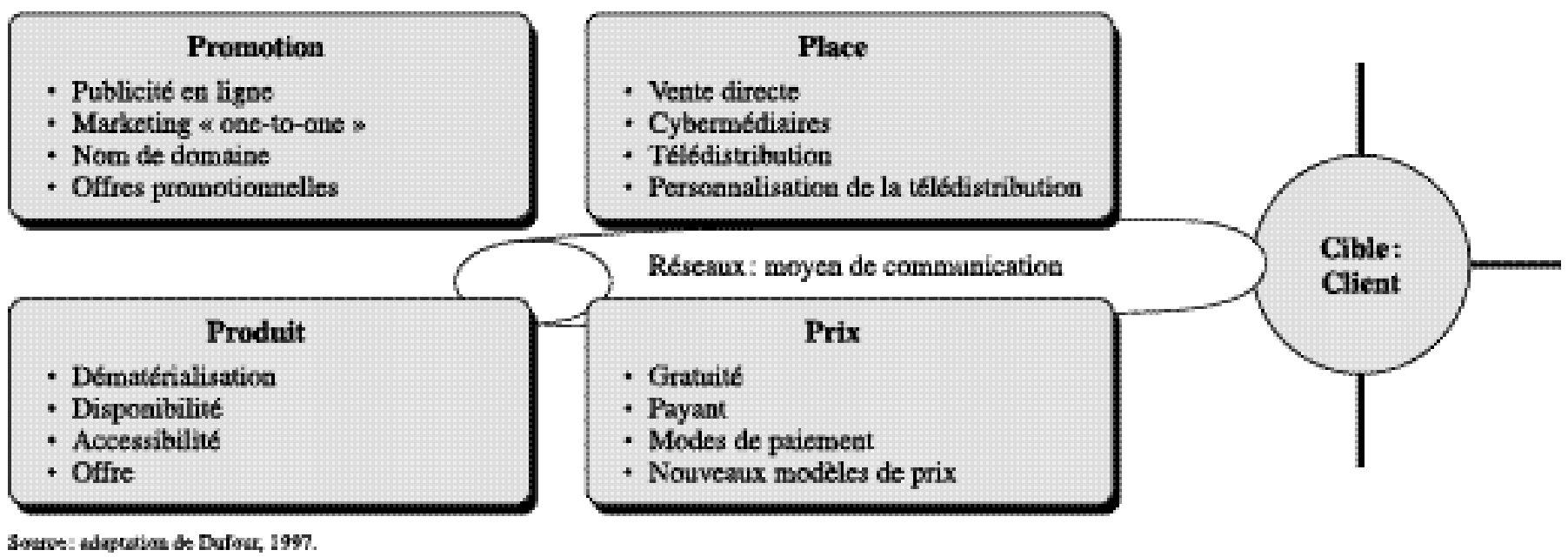

\section{Le produit}

En fait, il semble que le produit soit peu touché par son intégration dans le cyberespace. On trouve de tout sur Internet, et des biens de tous types s'échangent par le biais du commerce électronique, du livre jusqu'au vêtement, en passant par la commande d'épicerie (cas d'IGA $^{14}$ ) et l'automobile (Kotler ${ }^{15}$ rappelle qu'en 1998, plus de deux millions d'Américains ont acheté une voiture sans voir un seul garage). Il ne semble donc pas que le commerce électronique soit une barrière à la vente de quelque type de produit que ce soit. Ainsi, nous nous proposons d'analyser les caractéristiques spécifiques de ce média et de voir comment elles se rapprochent des caractéristiques existant pour les produits que l'on trouve dans le monde physique.

\section{La naissance de nouveaux produits}

Le concept de produit fait la distinction entre produit tangible et intangible. Si dans la réalité, ces deux notions sont bien distinctes et se présentent comme les bornes d'un continuum, il n'en demeure pas moins que l'Internet a intégré, dans certains cas, ces deux conceptions ${ }^{16}$. En effet, les documents sous forme électronique prennent de plus en plus de place dans ce monde, et le consommateur a désormais le choix entre une version papier ou une version électronique, dite dématérialisée. Dès 1997, l'OCDE faisait allusion à l'essor que pouvait prendre ce type de biens en précisant que "pour ces produits et d'autres biens immatériels - services audio, vidéo et d'information, services immobiliers et services aux entreprises (centres téléphoniques, services de paye et de facturation) -, l'impact économique du commerce électronique pourrait être fort et se faire sentir relativement vite». On note en effet aujourd'hui que ces services prennent une place de plus en plus importante, même si peu de consommateurs sont branchés à Internet, ce qui laisse entrevoir les opportunités de ce secteur ${ }^{17}$.

Les journaux électroniques, les rapports de recherche des universités ou de cabinets de consultants publics ou privés en sont les exemples mêmes. Le consommateur fait face à un dilemme: lire ses dépêches en temps réel à l'écran ou sur papier, et son choix est surtout lié à un besoin ponctuel.

Ceci ne signifie pas pour autant que la version papier traditionnelle des journaux soit en voie de disparition, mais seulement que ces derniers existent sous une forme plus commode et moins onéreuse pour l'organisation qui les rend disponibles et pour le consommateur qui les utilise. Citons à titre d'exemple que certains rapports de recherche sont disponibles sous deux formes; le client peut télécharger la plupart des rapports gratuitement en format $\mathrm{PDF}^{18}$ ou les commander, moyennant une facturation pour les coûts d'impression et les frais de port, et nonobstant un délai de livraison. Il est souvent nécessaire toutefois d'imprimer la version électronique pour la consulter plus aisément. Sa dématérialisation est donc utile uniquement pour sa conservation (elle est non altérable, demande moins d'espace de stockage) et son acheminement; elle nécessite souvent une remise en forme physique pour être utilisée plus efficacement. Cette version 
procure en outre des avantages comme la gratuité ou un coût réduit et un délai d'acheminement quasi instantané.

\section{La disponibilité du produit}

Virtualité ne rime pas forcément avec exhaustivité. Ainsi en ont décidé les entreprises «brick and $\operatorname{mortar}^{19}$ » qui, en développant un site Internet, ont offert à leurs clients, dans un premier temps, les produits les plus demandés. La boutique électronique de $\operatorname{Sears}^{20}$ ne propose que quelques centaines d'articles parmi les milliers de son catalogue et de ses magasins. De la même façon, Canadian Tire $^{21}$, qui permet depuis décembre 2000 seulement de magasiner en ligne, n'offre pas tous les articles présents en magasin.

Ce choix délibéré de ne proposer aux internautes qu'une sélection de produits se justifie par le fait que le marché est restreint: seulement $39 \%$ des ménages canadiens et $25 \%$ des ménages québécois étaient branchés à Internet en $2000^{22}$. Partant de ce fait, les modèles d'affaires électroniques ont dû prendre en compte une tendance pour distinguer disponibilité traditionnelle et virtuelle: la personnalisation de l'offre ${ }^{23}$. En effet, la personnalisation permet, après avoir identifié les besoins du consommateur, de lui proposer un produit répondant à ses caractéristiques (démographiques, profil de consommateur, etc.), permettant ainsi de faire du «sur mesure». Si Sears propose très peu de produits pour l'âge d'or, c'est parce que ce segment de marché ne représente pas un type de consommateur magasinant par la voie électronique.

\section{Les modèles d'affaires électroniques ont dû prendre en compte une tendance pour distinguer disponibilité traditionnelle et virtuelle : la personnalisation de l'offre.}

Finalement, ces géants de la distribution ont sans doute suivi les conseils de Benjamin et Wigand ${ }^{24}$, préconisant quelques tendances concernant les produits. Les auteurs ont en effet établi quelques prédictions, que nous reprenons ici pour la variable qui nous concerne. Ils précisent que les produits standards seront de plus en plus vendus électroniquement en raison du fait que les consommateurs n'ont pas besoin de les voir ni de les toucher. En extrapolant cette tendance, il y a fort à parier que les épiceries en ligne vont se développer au fur et à mesure de l'équipement des ménages. La femme active, gérant l'épicerie à la maison et désirant obtenir plus de temps libre, risque fort d'opter pour ce type de magasinage, lui permettant de se libérer de cette sacrosainte corvée.

Par ailleurs, les mêmes auteurs précisent que les produits nécessitant un complément d'information important se vendront mieux par les médias électroniques en raison du fait que les consommateurs peuvent obtenir un complément d'information directement sur le site du manufacturier. Ainsi, Sears correspond davantage à la première tendance, où les cyberconsommateurs font avant tout des achats répétés d'articles connus tandis que Canadian Tire s'inscrit dans ce deuxième adage, les produits plus techniques qu'ils proposent permettant au consommateur d'accéder à une information bien plus large que celle fournie par la vision subjective d'un seul vendeur.

\section{L'accessibilité du produit}

Deux éléments sont à considérer dans ce cadre : les biens et les services ${ }^{25}$. Concernant les biens, leur accessibilité n'est pas instantanée comme dans le cadre d'une transaction physique. L'achat d'un disque, par exemple, implique un délai lié à sa manutention, son emballage, son envoi et sa livraison, ce qui suppose une attente plus ou moins longue. Ainsi, le modèle traditionnel relationéchange-transaction s'inscrit dans un nouvel ordre dans lequel l'échange apparaît une fois que le marchand virtuel a débité le montant de la transaction. Le consommateur n'agit plus dans un univers «donnant-donnant», mais dans un contexte où il pose le premier geste: il paie avant de recevoir le produit correspondant à cette transaction.

\section{Le modèle traditionnel relation-échange- transaction s'inscrit dans un nouvel ordre dans lequel l'échange apparaît une fois que le marchand virtuel a débité le montant de la transaction.}

Certaines recherches démontrent que dans ce contexte, le consommateur n'est pas encore prêt à payer le même prix pour un article vendu dans Internet que pour un article vendu dans des commerces traditionnels, notamment lorsque ces deux produits sont du même prix et que dans le premier cas, il faille attendre plus longtemps avant de l'avoir en sa possession ${ }^{26}$. 
L'achat d'une information, au contraire, se fait souvent en temps réel, et le fait d'accepter de livrer son numéro de carte de crédit donne au consommateur la clé lui permettant d'accéder instantanément au contenu informationnel du site en question. Contrairement à une transaction physique, dans le cadre de laquelle la confirmation et l'autorisation d'accès à cette information sont obtenues par courrier, la voie électronique dispose d'un avantage supplémentaire.

\section{L'offre du produit}

Si le prix n'est pas un argument de positionnement, les marchands virtuels doivent également changer le paradigme classique d'offre et se concentrer davantage sur l'un des cinq avantages concurrentiels définis par Alba, Janiszewski, Lutz, Sawyer et Wood $^{27}$ : la logistique, la présentation des produits, l'originalité de l'offre, l'utilisation des informations clients et la composition de l'assortiment.

De ces cinq éléments, l'originalité de l'offre est celui que nous étudierons ici. En effet, le commerce électronique permet une symbiose entre produit et service. Il n'est qu'à penser au diagnostic personnalisé, aux services de réservation et d'assistance de nombreux hôtels regroupés sous la bannière Hôtellerie champêtre ${ }^{28}$ pour se rendre compte que ces éléments englobent le produit de telle façon qu'il est presque impossible de le considérer comme un service pur plutôt que comme le produit étendu. Le service rendu au consommateur est tellement étendu qu'il lui confère une connaissance quasi totale de ses besoins et ses désirs.

Ainsi, l'offre est à repenser dans son intégralité. D'ailleurs, selon Volle ${ }^{29}$, les produits proposés par voie électronique ne devraient pas s'articuler autour d'une logique de l'offre (ex: marque, famille de produits, etc.) mais plutôt selon une logique de consommation (ex: tous les produits susceptibles d'être consommés par tel individu).

\section{Les produits proposés par voie électronique ne devraient pas s'articuler autour d'une logique de l'offre (ex : marque, famille de produits, etc.) mais plutôt selon une logique de consommation (ex: tous les produits susceptibles d'être consommés par tel individu).}

L'auteur explique qu'il s'agit ici de la logique de «personal market place», où l'unité d'analyse n'est plus le produit en question mais l'individu, et précise que les nouveaux marchands «vont émerger autour de cette logique [...] leur compétence distinctive s'exprimera en terme de compréhension des clients, et ils reposeront sur des partenaires-fournisseurs maîtrisant exclusivement l'offre, mais qui n'auront pas de contact direct avec ces clients».

Il semble d'ailleurs que le vendeur de livres Amazon s'oriente à terme vers ce concept, et il est aisé d'imaginer par ce principe qu'un client achetant un livre sur la forêt tropicale, par exemple, se voie proposer toute une gamme de produits et de services relatifs à ce thème: expéditions-voyages en Amazonie, adhésion à des associations de défense de la forêt amazonienne, objets primitifs élaborés par les Indiens, etc.

Dans un autre ordre d'idées, un commerce comme Canadian Tire pourrait proposer à un acheteur de perceuse la gamme de forets et de vis, des lunettes de protection ainsi que la trousse pharmaceutique de premiers soins allant de pair avec le produit!

Nous pensons que l'avantage d'une telle démarche réside en l'amélioration de la compréhension de la logique de consommation. Il s'agit de comprendre pourquoi et comment le consommateur associe les objets qu'il achète les uns aux autres, et non plus pourquoi il choisit une référence par opposition à une autre.

\section{Les autres éléments constitutifs du produit}

Des éléments constitutifs du produit, deux en particulier sont à prendre en considération, avec un tantinet plus de respect que les autres, car ils entrent intégralement dans la stratégie marketing de l'entreprise. La marque d'une part, comme nous le verrons dans le paragraphe suivant, mais également le service attaché au produit (notion de produit augmenté voire potentiel) offrent une différenciation fondamentale pour toute entreprise intéressée par un affichage sur le Web.

\section{La marque}

«Qui veut créer une marque sur Internet ne doit pas considérer le Web comme un média, mais comme une activité » rappellent Ries \& Ries ${ }^{30}$. Il ne s'agit pas de croire que seules les marques ayant une forte reconnaissance dans le monde réel ont un «cyber-avenir». L'expé- 
rience montre que Yahoo !(tm) ${ }^{31}$ a bien plus de notoriété et attire plus d'internautes que les sites Web des principaux journaux d'information américains.

Ces deux auteurs énoncent d'ailleurs onze principes pour permettre à une marque de s'épanouir pleinement sur Internet et préconisent un changement de paradigme aux entrepreneurs de l'Internet. Les notions d'interactivité, d'unicité, de mondialisme et de temps ne sont pas étrangères aux succès des entreprises ayant intégré une stratégie Web. Lehu ${ }^{32}$ s'inscrit dans ce même courant de pensée en proposant une approche pratique (avec ses histoires d'horreur et ses cas de réussites exemplaires) pour permettre à une entreprise de réussir avec brio dans l'Internet.

\section{Le service et le SAV}

Talon d'Achille de ce moyen de communication, le service est une faille si l'on considère le conseil comme un élément de la vente. Bien évidemment, les modèles de comportement de consommateur proposent tous une phase de recherche d'information que le consommateur assouvit de lui-même ou en se référant à des spécialistes (les vendeurs, conseillers, etc.).

La virtualisation de ces derniers étant impossible en tant que telle, le magasinage électronique se fait sans conseiller $^{33}$; seule la description des produits ou du service peut servir de point de repère au consommateur. Passé ce cap, il n'a pas droit à d'autres renseignements, sauf, dans certains cas, la présence d'un phare utile matérialisé par la demande de renseignements complémentaires via une adresse de courrier électronique.

Certaines entreprises, comme Sony, misent sur ce type de service pour proposer au client un produit générique complet, et offrent un service en ligne : c'est ainsi qu'est né SOS (Sony Online Support). Comme le rapporte Kotler $^{34}$, la publicité de SOS précise ceci:

«Les ordinateurs ont beaucoup progressé, mais pas l'assistance... Jusqu'à ce que nous développions SOS (Sony Online Support). Le principe est simple: si vous avez un problème, nous vous aiderons à le résoudre. En fait, nous n'avons même pas besoin de votre aide. Avec votre permission, nous nous connecterons à votre ordinateur, nous l'examinerons et nous résoudrons le problème (même à des milliers de kilomètres de distance). Tout ce que vous avez à faire, c'est de cliquer sur notre petit icône et votre ordinateur appellera le centre de service. Nous ferons le reste.»

Ainsi, même à distance, les ingénieurs de Sony ont mis en place un système permettant de faire un suivi des produits achetés et allant ainsi de pair avec la tendance de vente des ordinateurs par correspondance. Dell s'est illustré par cette méthode, mais sa ligne téléphonique gratuite ne répond plus forcément aux exigences des consommateurs, sans cesse en demande d'un service de plus en plus complet.

Le service faisant partie intégrante du produit, un tel concept développé par Sony permet d'éviter bien des écueils mentionnés par Paasuraman, Zeithmal \& Berry ${ }^{35}$ dans leur modèle SERVQUAL. Un rapprochement du client par le délivrement d'un service de qualité est un atout supplémentaire pour une entreprise, d'autant plus qu'à l'instar des ventes dans le monde physique, le service est un élément de différenciation permettant de fidéliser le client. Et si lui vendre un produit est une chose, le fidéliser et vendre aux personnes qu'il recommande à l'entreprise est un autre défi.

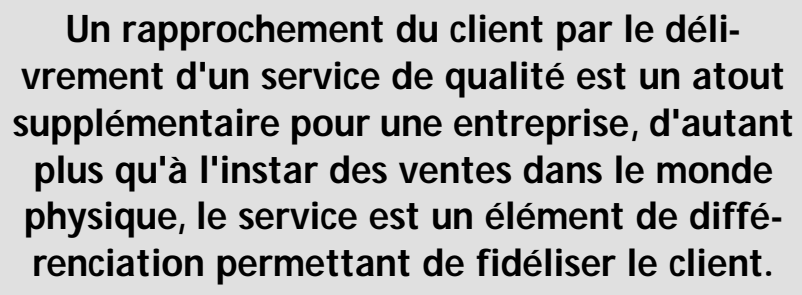

\section{Conclusion}

Dans les relations Business-to-Consumer, les six besoins « classiques» du consommateur (sécurité, orgueil, nouveauté, confort, argent, sympathie) sont systématiquement escamotés: on ne voit la plupart du temps que les catalogues mornes qui ne se sont donné qu'une seule mission: informer. Les dimensions interactionnelles et transactionnelles sont occultées, car les entreprises voient en Internet uniquement une nouvelle forme de promotion, et non un objet de faire des affaires à part entière. Ces données sont d'ailleurs confirmées par les résultats d'une étude menée par Bégin, Tchokogué et Boisvert ${ }^{36}$. Ayant étudié les sites Web de 2725 entreprises québécoises, les auteurs ont mis en valeur le fait que plus de $77 \%$ avaient un site promotionnel (informer); les sites transactionnels représentant moins de $10 \%$ du total de 
ces entreprises. Cet aspect (n'utiliser qu'une des variables du marketing-mix) constitue un défaut, d'autant plus renforcé lorsque les entreprises ne font pas les efforts nécessaires pour proposer un site à la hauteur du consommateur. Amami et Thévenot ${ }^{37}$ précisaient à cet égard que « la technologie Internet amène une menace et un défi considérables pour les entreprises existantes qui ont pris des décennies à bâtir leur produit de marque et leurs réseaux de distribution ». Les auteurs considèrent d'ailleurs qu'une stratégie marketing sur Internet en terme de produit se caractérise avant toute chose par une façon «d'innover et de répondre aux attentes des consommateurs».

\section{Amami et Thévenot précisent que « la technologie Internet amène une menace et un défi considérables pour les entreprises existantes qui ont pris des décennies à bâtir leur produit de marque et leurs réseaux de distribution».}

Ainsi, dans ce chaos généralisé que sont les NTIC pour de nombreuses entreprises, et notamment des PME, seuls parviennent à tirer leur épingle du jeu les marchands qui ont un produit sur lequel il existe un déficit d'information ou des produits qui séduisent par eux-mêmes. Notons à cet égard le cas de réussite exemplaire de la PME québécoise Arontec ${ }^{38}$, spécialisée dans la construction de maisons en queues d'aronde.

Il n'existe pas de réponse unique aux entreprises se demandant quel modèle utiliser pour présenter leurs produits; il n'y a que des questions à se poser pour tout dirigeant désireux de s'implanter sur le Web. Aucun modèle ne saurait avoir la prévalence dans un secteur d'activité. Ce scénario idéaliste, vanté par les chantres des industries du logiciel et de l'informatique, ne prenait pas en compte les particularités des entreprises, leurs objectifs, ni la façon dont elles pouvaient (et non devaient) intégrer Internet dans leur stratégie de marketing. Comme nous venons de le voir, le cybermix dont le produit fait partie intégrante ne répond pas à toutes les questions stratégiques d'une entreprise. D'un autre côté, c'est grâce à un marketing très orienté qu'Arontec a pu attirer des clients des quatre coins du monde.

\section{Notes et références}

1 William Menvielle est étudiant au doctorat en administration des affaires, Denis Pettigrew est professeur de marketing et Jocelyn D. Perreault est professeur de marketing. Ils exercent leurs fonctions à l'Université du Québec à Trois-Rivières.
2 PALLAB, P. (1996). «Marketing on the Internet», Journal of Consumer Marketing, vol. 13, $\mathrm{n}^{\circ}$ 4, p. 27-39.

3 LUEDI, A. F. (1997). «Personalize or Perish», Electronic Markets, vol. $7, \mathrm{n}^{\circ} 3$.

4 CRONIN, Mary J. et autres (1994). Faire affaire sur Internet : un guide pour gestionnaires, adaptation française de Doing more business on the Internet, Guérin Universitaire, Montréal. (1998 pour la traduction française).

5 SETTLES, C. (1995). Cybermarketing: Essentials for success, Emeryville, Californie, Ziff-Davis Press, 275 p.

6 KEELER (1995). Cybermarketing, AMCAOM (American Management Association).

7 SETTLES, C. (1995). Op. cit., note 5.

8 Si l'on comparait Internet à une autoroute - on compare d'ailleurs souvent ce réseau mondial à une autoroute d'information -, les modèles d'affaires seraient les différents véhicules capables d'emprunter cette autoroute.

9 DUFOUR, A. (1997). Le cybermarketing: intégrer Internet dans la stratégie d'entreprise, Presses universitaires de France, Paris. (Collection Que sais-je?).

10 Pour plus de renseignements à ce propos, le lecteur pourra étancher sa soif de curiosité en lisant l'article de TIMERS, Paul (1998). «Business Models for Electronic Markets», Electronic Markets, vol. 8, $\mathrm{n}^{\circ} 2$.

11 PEPPERS, D. and M. ROGERS (1993). The One-to-One Future : Building Relationships One Customer at a Time, Currency Doubleday.

12 KOTLER, P., P. FILIATRAULT et R. E. TURNER (2000). Le management $d u$ marketing ( $2^{\mathrm{e}}$ éd.), Gaëtan Morin Éditeur, Boucherville, Québec.

13 DUFOUR,A. (1997). Op. cit., note 9.

14 Voir http://www.iga.net

15 KOTLER, P. (1999). Le Marketing selon Kotler, Éditions Village Mondial, Paris.

16 BAKOS, Y. (1998). The emerging role of electronic marketplaces on the Internet, Communications of the ACM.

17 On peut véritablement parler de nouveau secteur d'activité depuis que les Nord-Américains ont généré ce nouveau secteur d'activité sous la rubrique «Industrie des produits informationnels et culturels».

18 «Portable Document Format», type de fichier nécessitant le logiciel Acrobat Reader pour être lu, reconnu indifféremment par tout type de système Macintosh ou PC.

19 Littéralement «brique et mortier», en référence aux entreprises traditionnelles ayant un magasin physique.

20 Voir http://www.sears.ca

21 Voir http://www.canadiantire.ca

22 POUSSART, B. et E. LACROIX (2000). Le commerce électronique, CEFRIO, Isabelle Quentin Éditeur, Montréal. (Collection Infomètre). 
23 BAKOS, Y. (1998). Op. cit., note 16.

24 BENJAMIN, R. and R. WIGAND (1995). «Electronic markets and virtual value chains on the information Superhighway », Sloan Management Review, hiver.

25 Peu d'études se sont intéressées véritablement aux enjeux des modifications subies par les produits et les services sur Internet. Une piste de recherche consisterait à étudier la classification des produits de Aspinwall ou Copeland, l'analyse des services de Eiglier et Langeard sur ce nouveau médium.

26 KOTLER, P. (1999). Op. cit., note 15.

27 ALBA, J. et autres (1997). «Interactive Home shopping : Consumer, Retailer and Manufacturer Incentives to Participate in Electronic Marketplaces », Journal of Marketing, vol. 61, p. 38-53.

28 Voir http://www.hotelleriechampetre.com

29 VOLLE, P. (1999). «Du marketing des points de vente à celui des sites marchands: spécificités, opportunités et questions de recherche», Cahier de Recherche ${ }^{\circ}$ 276, Centre de Recherche DMSP, Université Dauphine, Paris.

30 RISE,A. et L. RIES (2001). Les 11 lois de la e-marque, Dunod, Paris.
31 Voir http://www.yahoo.com

32 LEHU, J.-M. (2001). Concevoir, protéger et gérer la marque sur l'Internet, [stratégiesdemarque.com], Éditions d'Organisation, Paris.

33 C'est d'ailleurs ce qui peut constituer un avantage si l'on considère que certains clients n'aiment pas magasiner en raison d'un vendeur trop accaparant (Grégoire et Nantel, 1998).

34 KOTLER, P. (1999). Op. cit., note 15.

35 PAASURAMAN, A., V. SEITHMAL and L. BERRY (1985). «A conceptual model of Service Quality and its implications for future Research », Journal of Marketing, automne.

36 BÉGIN, L., A. TCHOKOGUÉ et H. BOISVERT (2001). Pour un déploiement stratégique du commerce électronique, Isabelle Quentin Éditeur.

37 AMAMI, M. et J. THÉVENOT (2000). «L'Internet marchand: caractérisation et positionnement stratégiques», Systèmes d'information et Management, vol. 5, $\mathrm{n}^{\circ} 1$.

38 Voir http://www.arontec.ca 